\title{
Eletrólise com radiofrequência no tratamento da triquíase
}

\author{
Treatment of trichiasis with high-frequency radio wave electrosurgery
}

\author{
Rodrigo Beraldi Kormann ${ }^{1}$ \\ Hamilton Moreira ${ }^{2}$
}

Trabalho realizado no Hospital de Olhos do Paraná e no Hospital Universitário Evangélico de Curitiba Curitiba (PR) - Brasil.

${ }^{1}$ Chefe do Serviço de Oculoplástica da Universidade Evangélica de Curitiba - Curitiba (PR) - Brasil.

${ }^{2}$ Professor Titular do Hospital Universitário Evangélico de Curitiba; Professor Adjunto da Universidade Federal do Paraná - UFPR - Curitiba (PR) - Brasil.

Endereço para correspondência: Rua Deputado Emilio Carlos, 50 - Apto 52 - Curitiba (PR) CEP 80540080

E-mail: robk@bk.brtdata.com.br

Recebido para publicação em 07.05.2006

Última versão recebida em 02.09.2006

Aprovação em 12.09.2006

Nota Editorial: Depois de concluída a análise do artigo sob sigilo editorial e com a anuência do Dr. José Américo Bonatti sobre a divulgação de seu nome como revisor, agradecemos sua participação neste processo.

\begin{tabular}{|l|}
\hline RESUMO \\
\hline Objetivos: Triquíase é uma condição adquirida dos cílios, que perdem o \\
direcionamento normal e tocam a superfície ocular. O tratamento da \\
triquíase tem sido um problema terapêutico. Apesar das várias modali- \\
dades de tratamento: epilação mecânica, eletrólise com bipolar, crioterapia, \\
fotocoagulação com laser de argônio e procedimentos cirúrgicos, a \\
recidiva da triquíase é comum. Com o objetivo de encontrar técnica \\
próxima ao ideal, realizamos estudo utilizando eletrólise com aparelho de \\
radiofreqüência, avaliando-se a efetividade da técnica. Métodos: Foram \\
estudados, prospectivamente, 34 pacientes (54 segmentos palpebrais) \\
com triquíase, submetidos à eletrólise com aparelho de radiofrequiência. \\
Reavaliaram-se os pacientes em oito semanas, quanto à cura, recidiva e \\
possíveis complicações. Resultados: Observou-se a cura da triquíase \\
com apenas uma única sessão de eletrólise com radiofreqüência em 22 \\
pacientes e com duas ou mais sessões em 12 pacientes. Conclusão: A \\
eletrólise com aparelho de radiofreqüência é método simples, de baixo \\
custo, que não demanda experiência do cirurgião, com ausência de \\
complicações relevantes e com alto índice de sucesso.
\end{tabular}

Descritores: Pestana/patologia; Remoção de cabelo; Eletrólise/utilização; Eletrocirurgia; Recidiva

\section{INTRODUÇÃO}

Triquíase é uma condição adquirida dos cílios, normalmente posicionados na lamela anterior palpebral, que perdem o direcionamento normal e tocam a superfície ocular.

É uma doença comum de etiologia variada, sendo as mais comuns: blefarite, tracoma, queimaduras, trauma, cirurgia prévia e doenças cicatriciais como penfigóide ocular e síndrome de Stevens Johnson.

Pelo trauma constante dos cílios à superfície ocular, estão presentes sintomas de lacrimejamento, sensação de corpo estranho, blefaroespasmo reflexo, podendo causar erosões corneanas, infecção, vascularização e opacificações, com risco real de baixa da acuidade visual.

A triquíase é classificada em menor ou maior, conforme a quantidade de cílios mal direcionados. A triquíase é dita maior, quando cinco ou mais cílios estão acometidos. Um método para se avaliar a triquíase é quantificando-se o número de segmentos palpebrais envolvidos: terço nasal, central e temporal, facilitando a avaliação no pós-operatório.

O tratamento da triquíase tem sido um problema terapêutico. Apesar das várias modalidades de tratamento, a recidiva da triquíase é comum ${ }^{(1-2)}$. Temporariamente podemos usar lubrificantes, lente de contato terapêutica e epilação mecânica, que pode ser realizada pelo próprio paciente ou pelo mé- 
dico. Quanto aos tratamentos definitivos, existem vários: eletrólise com aparelho bipolar, eletrólise com aparelho de radiofreqüência, crioterapia, o uso de laser de argônio e procedimentos cirúrgicos, cada um com suas vantagens e desvantagens.

Ainda não se encontrou o método ideal, com efetividade completa, facilidade na execução, de baixo custo e sem complicações. A eletrólise com aparelho bipolar é dolorosa, necessita de infiltração anestésica local e tem alta recorrência ${ }^{(1)}$. A crioterapia é um método com bons resultados, mas com grande potencial de complicações. A técnica de ablação dos cílios triquiáticos com fotocoagulação com laser de argônio, provavelmente é a que tenha chegado mais perto atualmente do ideal, pois possui uma alta eficácia com o mínimo de complicações, no entanto o aparelho de laser é de alto custo, a técnica exige treinamento e os parâmetros ainda não estão padronizados.

Com o objetivo de encontrar uma técnica próxima do ideal, realizamos um estudo no tratamento da triquíase, utilizando eletrólise com aparelho de radiofreqüência, avaliando-se a efetividade da técnica.

\section{MÉTODOS}

Foram estudados, prospectivamente, 34 pacientes (54 segmentos palpebrais) com triquíase, atendidos nos ambulatórios de Plástica Ocular da disciplina de Oftalmologia do Hospital de Olhos do Paraná e Hospital Universitário Evangélico de Curitiba, entre os meses de março a dezembro de 2004, submetidos a eletrólise com radiofrequiência (Aparelho Wavetronic 5000 LLEP Master), por um único cirurgião. Alguns pacientes haviam realizado outros tratamentos para a afecção em estudo, que serão citados. Todos os pacientes autorizaram por escrito o procedimento através do termo de esclarecimento e livre consentimento.

Os pacientes foram avaliados quanto ao sexo, idade, etiologia da triquíase e tratamentos anteriores. Após ter sido diagnosticado a triquíase, o paciente foi avaliado conforme os critérios de inclusão (triquíase menor ou maior, ausência de lesões palpebrais, entrópio ou ectrópio e que não estivessem na fase aguda da doença). Anotou-se o olho acometido (direito e/ou esquerdo), a pálpebra envolvida (superior e/ou inferior) e o segmento palpebral (nasal, central, temporal).

Agendado o procedimento cirúrgico, orientou-se ao paciente que não removesse os cílios triquiáticos (epilação mecânica) no mínimo trinta dias que antecedesse a data da cirurgia, se o incômodo fosse grande, adaptávamos uma lente de contato terapêutica. A eletrólise com aparelho de radiofrequiência foi realizada em ambiente cirúrgico, com paciente deitado, sob todas as condições de assepsia necessárias e com anestesia local xilocaína $2 \%$ associado à epinefrina a 1:100.000, próximo à margem palpebral afetada e com microscópio cirúrgico.

O aparelho de radiofreqüência (Wavetronic 5000 LLEP Master) foi usado com os parâmetros em 50\% de corte e 50\% de coagulação e potência de $1 \mathrm{mV}$. A ponteira utilizada foi a específica para eletrólise e a placa neutra em contato direto com a pele do paciente, próximo da cabeça, conforme solicitações técnicas por parte da fornecedora do aparelho. A ponteira era introduzida em posição co-axial ao folículo piloso triquiático, 3 a $4 \mathrm{~mm}$ de profundidade. O tempo de aplicação baseava-se na alteração do tecido superficial (variando de um a dois segundos), retirava-se a ponteira e quando o cílio não vinha junto, tirávamos com uma pinça de ponto, sem fazer força, se houvesse resistência na remoção do cílio tratado, nova aplicação era realizada.

No pós-operatório orientou-se colírio de antibiótico e corticóide por sete dias e analgésico via oral. O retorno foi agendado em oito semanas após a cirurgia, lembrando ao paciente que não removesse eventual cílio triquiático que viesse a aparecer neste período. Outros retornos ou nova sessão de tratamento eram agendados quando necessário. Durante o retorno observou-se à biomicroscopia na lâmpada de fenda a presença ou não de cílios triquiáticos. Os pacientes foram submetidos até três sessões de eletrólise com radiofrequiência, de acordo com a recidiva. Todos os pacientes continuaram sendo acompanhados ambulatorialmente e orientados a retornar diante de qualquer sintoma de recorrência.

De acordo com a natureza dos dados estudados, procedeu-se ao tratamento estatístico julgado adequado. O mesmo constou da elaboração de quatro tabelas e um gráfico. Para as variáveis acima apresentadas calcularam-se as freqüências: segmentos palpebrais e resultados do primeiro tratamento. Aplicou-se o teste z para diferença de proporções, com nível de significância $p<0,05$.

\section{RESULTADOS}

Dos 34 pacientes estudados, 21 eram do sexo feminino $(61,76 \%)$ e 13 do sexo masculino $(38,23 \%)$. A idade dos pacientes variou de 31 a 84 anos, sendo a média de 64,88 $\pm 12,94$.

Quanto à etiologia, 21 pacientes $(61,76 \%)$ tiveram a causa desconhecida, 6 pacientes blefarite, 4 cirurgia ocular prévia, 2 tracoma e 1 queimadura por cal (Gráfico 1).

Em relação ao tratamento anterior realizado, 15 pacientes

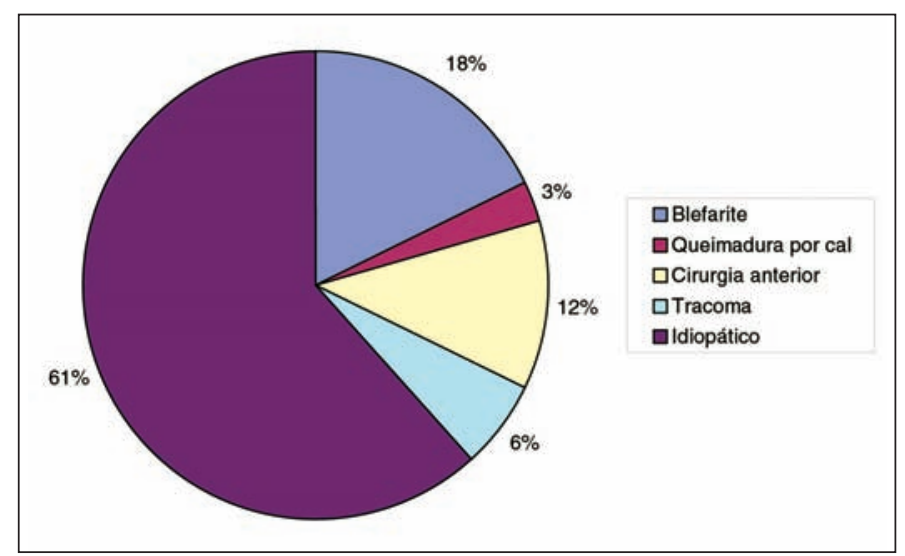

Gráfico 1 - Distribuição por etiologia - 34 pacientes 
$(44,12 \%)$ nunca haviam tratado, 14 pacientes $(41,18 \%)$ já havia epilado os cílios triquiáticos e 5 pacientes $(14,7 \%)$ tinham realizado cirurgia prévia.

O olho direito foi acometido em 20 pacientes e o olho esquerdo em 24 pacientes, sendo que em 10 pacientes eram ambos os olhos afetados.

As pálpebras inferiores (27 pálpebras) foram mais acometidas que as pálpebras superiores (13 pálpebras), sendo que o mais afetado na pálpebra inferior era o segmento palpebral central $(85,71 \%)$ e na pálpebra superior o segmento palpebral temporal $(57,89 \%)$ (Tabelas 1 e 2).

Observou-se a cura da triquíase com apenas um tratamento de eletrólise com radiofreqüência em 22 pacientes e com dois ou mais tratamentos em 12 pacientes (Tabela 3). Quanto aos segmentos palpebrais, 42 foram curados com um único tratamento de eletrólise com radiofrequiência e 12 segmentos palpebrais foram curados com dois ou mais tratamentos (Tabela 4).

As complicações limitaram-se à recidiva do cílio triquiático e um caso de tilose (espessamento da margem palpebral).

\section{DISCUSSÃO}

A triquíase é uma condição comum, que cronicamente pode causar alterações na superfície ocular, levando à baixa de acuidade visual.

A triquíase possui uma complexidade de problemas terapêuticos ${ }^{(3)}$. O uso de lubrificantes oculares proporciona alívio temporário e a lente de contato terapêutica ajuda provisoriamente, sendo problema para os pacientes com olho seco e pelo custo.

As técnicas para correção da triquíase são variadas, mas todas com o mesmo objetivo: eliminar o(s) cílio(s) triquiático(s) definitivamente e com ausência de complicações.

A epilação é um método simples, de baixo custo, relativamente livre de complicações, pode ser realizada pelo próprio paciente ou pelo médico, mas o problema é a recorrência, sendo considerada um tratamento temporário(4).

A eletrólise com aparelho bipolar é mais utilizada para triquíase focal, a porcentagem de sucesso varia, o procedimento pode ser desconfortável para o paciente e induzir a deformidades marginais ${ }^{(4)}$. A eletrólise necessita de infiltração anestésica local e tem alta recorrência, devido a agulha ser direcionada erroneamente a base do folículo piloso ${ }^{(1)}$.

A despigmentação da pele palpebral é bem conhecida complicação da crioterapia para triquíase. A despigmentação da pele palpebral ocorre, pois os melanócitos são destruídos na temperatura necessária para o tratamento dos cílios triquiáticos $^{(5)}$. Estudos clínicos em humanos confirmaram ser preciso temperaturas menores que $-15^{\circ} \mathrm{C}$ para o sucesso da ablação dos cílios ${ }^{(6-9)}$. A crioterapia tem sucesso no tratamento dos cílios aberrantes em $90 \%$ ou mais. O congelamento pode incitar cicatrização e algumas vezes produzir entrópio, particularmente em pacientes com desordem inflamatória, tal como penfigóide ocular e herpes zoster oftálmico ${ }^{(10)}$. A crioterapia

\begin{tabular}{|lcc|}
\hline \multicolumn{3}{|c|}{ Tabela 1. Distribuição por idade } \\
\hline Idade & $\mathbf{f}$ & $\%$ \\
$31-40$ & 3 & 8,82 \\
$41-50$ & 3 & 8,82 \\
$51-60$ & 3 & 8,82 \\
$61-70$ & 12 & 35,29 \\
$71-80$ & 11 & 32,35 \\
81 ou mais & 2 & 5,90 \\
Total & 34 & 100 \\
\hline
\end{tabular}

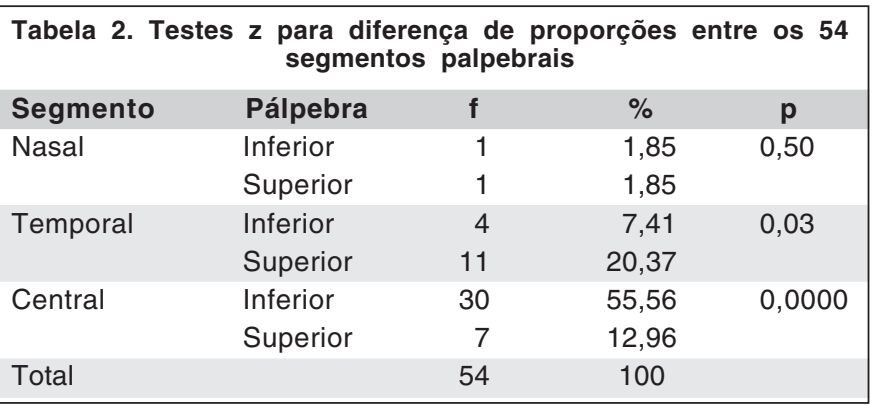

\begin{tabular}{lccc|}
\hline Tabela 3. Teste $\mathbf{z}$ para diferença de proporções do & $\mathbf{1}$ - \\
nos & $\mathbf{3 4}$ pacientes & \\
Pacientes & $\mathbf{f}$ & $\%$ & $\mathbf{p}$ \\
Curados & 22 & 64,71 & 0,004 \\
Não curados & 12 & 35,29 & \\
Total & 34 & 100 & \\
\hline
\end{tabular}

\begin{tabular}{|c|c|c|c|}
\hline Segmentos & $f$ & $\%$ & p \\
\hline Curados & 42 & 77,78 & 0,0000 \\
\hline Não curados & 12 & 22,22 & \\
\hline Total & 54 & 100 & \\
\hline
\end{tabular}

esta contra-indicada nos casos de penfigóide ocular, pela possibilidade de recidiva da doença ${ }^{(11)}$. As complicações da crioterapia estão em torno de $18 \%{ }^{(12)}$, incluindo: área de necrose, úlcera de córnea, aceleração de simbléfaro, hipopigmentação de pele, xerose, celulite e ativação de herpes zoster.

A vantagem do laser de argônio em relação à crioterapia é a reduzida inflamação, devido a precisa destruição tecidual ${ }^{(10)}$. A fotocoagulação com laser de argônio resulta da transformação de energia luminosa em energia térmica, através da absorção da luz pelo pigmento, com a modificação das proteínas teciduais ${ }^{(2,13)}$. A laserterapia é de difícil execução em pacientes não cooperativos, pacientes com tremores e impossibilidade de execução em pacientes acamados; outra desvantagem é o alto custo do aparelho. Dentre as vantagens podemos destacar a seletividade do procedimento ${ }^{(2)}$, dano tecidual mínimo dificilmente causando complicações ${ }^{(14)}$, alto índice de sucesso 
e baixa incidência de recorrência ${ }^{(17-18)}$. O sucesso com tratamento com laser de argônio foi de $59 \%$ com um tratamento e $70 \%$ com dois tratamentos ${ }^{(10)}, 45 \%$ de sucesso com um tratamento e $90,3 \%$ com dois tratamentos ${ }^{(15)}, 33 \%$ com um único tratamento e mais de $80 \%$ sucesso com mais de três sessões ${ }^{(16)}$. O laser de argônio destrói o folículo piloso focalmente em coelhos, com somente o mínimo de inflamação secundária nos tecidos adjacentes ${ }^{(4)}$. O laser de argônio possui efetividade de $100 \%$ e o mínimo de complicações ${ }^{(4)}$.

Outras técnicas cirúrgicas, como a cirurgia de Van Millengen (exérese da região com cúlios triquiáticos e enxerto de mucosa labial), são utilizadas quando existem vários cílios triquiáticos adjacentes no mesmo segmento palpebral, com bons resultados, mas sendo um procedimento cirúrgico complexo e para casos bem selecionados. A técnica cirúrgica precisa ser considerada no tratamento da triquíase, especialmente nos pacientes com doenças cicatriciais ${ }^{(17)}$.

A eletrocirurgia é o processo de corte/coagulação de tecido utilizando uma corrente alternada de alta frequiência ${ }^{(18)}$. A passagem da onda eletromagnética através do tecido biológico, em alta frequiência $(300 \mathrm{kHz}$ a $3,3 \mathrm{MHz})$, causa aceleramento das partículas iônicas intracelulares, desidratando as células quando a temperatura está em torno de $75^{\circ} \mathrm{C}$ e evaporando-as quando a temperatura está acima de $100^{\circ} \mathrm{C}$. A frequiência pode ser adaptada para diversas funções, como corte de tecidos, coagulação, morte da célula com manutenção de sua estrutura e volatização celular ${ }^{(19)}$.

Utilizado o aparelho de radiofrequência (Ellmann Surgitron), no modo corte/coagulação com potência de $1 \mathrm{mV}$. Foram tratados pacientes com entrópio e ectrópio e depois realizado remoção em bloco da pálpebra e esta enviada para exame anatomopatológico. As ondas de radiofreqüência promoveram destruição selecionada dos folículos pilosos, apenas os diagnosticados como triquiáticos, somente pequeno tecido adjacente foi alterado (menos de $0,5 \mathrm{~mm}$ da aplicação), impedindo deformidades marginais. Devido à baixa voltagem e alta amperagem da corrente e o tecido em contato com o eletrôdo por um curto período de tempo, há pouco calor lateral, causando o mínimo de dano à margem palpebral ${ }^{(17)}$.

Neste estudo preconizou-se ponteira padrão para eletrólise e os parâmetros utilizados baseados em estudo anterior ${ }^{(17)}$, com $50 \%$ corte e $50 \%$ coagulação, com potência de $1 \mathrm{mV}$. A profundidade foi de 3 a $4 \mathrm{~mm}$ e o tempo de aplicação variou de 1 a 2 segundos. O número de cílios triquiáticos não foi parâmetro de inclusão ou exclusão neste estudo, levando-se em conta estudos anteriores ${ }^{(17)}$, onde a destruição de tecido adjacente é mínima. Foi optado pela anestesia local com xilocaína $2 \%$ associada à epinefrina a 1:100.000, pois existem trabalhos que mostram preferência pela anestesia local em relação a anestesia tópica, em cerca de $81 \%$ dos pacientes ${ }^{(11)}$.

Em nosso estudo houve maior número de pacientes do sexo feminino $(61,76 \%)$ em relação ao sexo masculino $(38,23 \%)$ e a idade média obtida foi de 64,88 anos com desvio padrão de $\pm 12,94$, concordando com estudos anteriores ${ }^{(6,8,15)}$.

Quanto à etiologia, 21 pacientes $(61,76 \%)$ foram designa- dos ao grupo de causa idiopática, casos que se desconhecia ou não era comprovada a causa da triquíase, resultado semelhante ao obtido em 1991, onde 27 pacientes $(61,36 \%)$ tinham etiologia indefinida ${ }^{(10)}$. Ainda em nosso estudo, 6 pacientes tiveram como causa blefarite, 4 pacientes história de cirurgia prévia, 2 pacientes com diagnóstico clínico de tracoma e 1 paciente com queimadura por cal, concordando com a literatu$\mathrm{ra}^{(1,10-11,20)}$, onde estas são as principais causas e discordando em trabalhos ${ }^{(11,21)}$ onde a causa principal foi o tracoma. Devemos relevar a época e a região onde o estudo foi realizado. Tivemos uma paciente com síndrome de Stevens Johnson, mas que perdeu seguimento. Não existiram pacientes com diagnóstico de penfigóide ocular e nem de herpes zoster, outras causas freqüentemente descritas na literatura.

Em relação a tratamentos prévios, 15 pacientes $(44,12 \%)$ nunca haviam realizado qualquer tipo de tratamento, 14 pacientes $(41,18 \%)$ referiram epilação mecânica própria, familiar ou médico, cinco pacientes $(14,7 \%)$ outros procedimentos cirúrgicos variados, sendo uma eletrólise com aparelho bipolar. Nenhum paciente havia sido submetido a tratamento com crioterapia ou fotocoagulação com laser de argônio.

Quanto ao olho acometido, 24 pacientes tiveram o olho esquerdo afetado e 20 pacientes o olho direito, sendo que destes, 10 pacientes tinham os dois olhos comprometidos. As pálpebras inferiores foram acometidas com maior freqüência que as pálpebras superiores: 27 pálpebras inferiores e 13 pálpebras superiores, sendo que em 6 pacientes ambas as pálpebras eram afetadas.

Para uma melhor avaliação, cada pálpebra foi dividida em três segmentos palpebrais: nasal (terço nasal), central (terço central), temporal (terço temporal), pois em caso de triquíase no pós-operatório, nos facilitaria estudar a possibilidade deste cílio triquiático não ter sido tratado, se ele estiver presente num novo segmento palpebral. Avaliamos a freqüência de acometimento de tais segmentos, encontrando uma diferença estatisticamente significativa, comparando-se as pálpebras superior e inferior em relação aos segmentos palpebrais centrais $(85,71 \%$ na pálpebra inferior) e temporais $(57,89 \%$ na pálpebra superior) (Tabela 2). Não encontramos justificativa para tal resultado.

$\mathrm{Na}$ avaliação da cura, subdividimos em cura do paciente e cura por segmento palpebral. Dos 34 pacientes do estudo, todos os pacientes foram curados, necessitando de uma a três sessões de eletrólise com radiofreqüência, onde 22 pacientes $(64,71 \%)$ foram curados com um único tratamento $(\mathrm{p}<0,04)$, resultado relevante se comparado a outros trabalhos, onde a cura com um único tratamento foi de $42,1 \%^{(11)}, 27,2 \%^{(3)} \mathrm{e}$ $45 \%{ }^{(10)}$ com laser de argônio. Neste mesmo trabalho obtiveram $90,3 \%$ de sucesso com mais de duas sessões do tratamento, no caso laserterapia, enquanto nosso sucesso com dois ou mais tratamentos foi de $100 \%$ (Tabela 3 ).

O sucesso em relação aos segmentos palpebrais, também foi estatisticamente significativo ( $\mathrm{p}<0,0000)$, pois $77,78 \%$ dos segmentos palpebrais foram curados com um único tratamento (Tabela 4). Podemos explicar tal resultado, por alguns seg- 
mentos palpebrais terem menos cílios triquiáticos que outros e quanto menor o número de cílios triquiáticos por pálpebra, maiores são as chances de sucesso com menos sessões ${ }^{(3)}$.

Como complicações, citamos casos de insucesso com o primeiro tratamento e um paciente que apresentou espessamento discreto da margem palpebral (tilose), sendo este paciente portador de dez cílios triquiáticos num mesmo segmento palpebral, necessitando de duas sessões para a cura da triquíase. Não foi considerado como complicação, edema, eritema, hematoma palpebral apresentados no pós-operatório imediato.

A eletrólise com aparelho de radiofrequiência é um método simples, de baixo custo, que não demanda experiência do cirurgião, com ausência de complicações relevantes e com alto índice de sucesso.

\section{ABSTRACT}

Purpose: Trichiasis is an acquired condition in which the eyelashes are directed posteriorly, touching the surface of the cornea. It poses a complex therapeutic problem. Despite several therapeutic options (epilation, electroepilation, cryotherapy, laser ablation and standard surgical techniques), relapse is frequent. Methods: A prospective, nonmasked study was conducted to determine the efficacy of high-frequency radioelectrosurgery for treatment of trichiasis. Thirty-four patients (fiftyfour eyelid segments) were treated with this technique. After eight weeks, all patients were reviewed regarding the success rate, relapse and complications. Results: Cure was achieved after only one session of treatment in twenty-two patients, and two or more sessions in the remaining twelve. No complications have been reported. Conclusions: We consider high-frequency radioelectrosurgery a simple, not expensive, effective and free of complications technique for the treatment of trichiasis.

Keywords: Eyelashes/pathology; Hair removal; Electrolysis/ utilization; Electrosurgery; Recurrence

\section{REFERÊNCIAS}

1. Oshry T, Rosenthal G, Lifshitz T, Shani L, Yassur Y. Argon green laser photoepilation in the treatment of trachomatous trichiasis. Ophthal Plast Reconstr Surg. 1994;10(4):253-5.

2. Dantas RRA. Triquíase: fotocoagulação com laser argônio. Rev Bras Oftalmol. 1992;51(4):9-12.

3. Ladas ID, Karamaounas N, Vergados J, Damanakis A, Theodossiadis GP. Use of argon laser photocoagulation in the treatment of recurrent trichiasis: long-term results. Ophthalmologica. 1993;207(2):90-3.

4. Yung CW, Massicotte SJ, Kuwabara T. Argon laser treatment of trichiasis: a clinical and histopathologic evaluation. Ophthal Plast Reconstr Surg. 1994; 10(2):130-6.

5. Zabriskie NA, Nordlund JJ, Nerad JA. Unusual skin depigmentation following eyelid cryosurgery. Ophthal Plast Reconstr Surg. 1996;12(4):296-8.

6. Sullivan JH, Beard C, Bullock JD. Cryosurgery for treatment of trichiasis. Am J Ophthalmol. 1976;82(1):117-21.

7. Sullivan JH, Beard C, Bullock JD. Cryosurgery for treatment of trichiasis. Trans Am Ophthalmol Soc. 1976;74:189-202.

8. Wood JR, Anderson RL. Complications of cryosurgery. Arch Ophthalmol. 1981;99(3):460-3.

9. Johnson RL, Collin JR. Treatment of trichiasis with a lid cryoprobe. Br J Ophthalmol. 1985;69(4):267-70.

10. Bartley GB, Lowry JC. Argon laser treatment of trichiasis. Am J Ophthalmol. 1992;113(1):71-4.

11. Fonseca Junior NL, Lucci LMD, Paulino LV, Rehder JRCL. O uso do laser de argônio no tratamento da triquíase. Arq Bras Oftalmol. 2004;67(2):277-81.

12. Wood JR, Anderson RL. Complications of cryosurgery. Arch Ophthalmol. 1981;99(3):460-3.

13. Hata MM, Monteiro ECL, Schellini SA, Aragon FF, Padovani CR. Laser de argônio no tratamento da triquíase e da distiquíase. Arq Bras Oftalmol. 1999;62(3):285-95.

14. Sharif KW, Arafat AF, Wykes WC. The treatment of recurrent trichiasis with argon laser photocoagulation. Eye. 1991;5(Pt 5):591-5.

15. Awan KJ. Argon laser treatment of trichiasis. Ophthalmic Surg. 1986;658-60.

16. Campbell DC. Thermoablation treatment for trichiasis using the argon laser. Aust NZ J Ophthalmol. 1990;18:427-30.

17. Hurwitz JJ, Johnson D, Howarth D, Molgat YM. Experimental treatment of eyelashes with high-frequency radio wave electrosurgery. Can J Ophthalmol. 1993;28(2):62-4.

18. Fundamentos físicos da cirurgia de alta frequiência. Manual do aparelho Wavetronic 5000 LLEP Master. São Paulo: Loktal Medical Electronics; sd.

19. Eduardo Paulino. Radiofrequência multifuncional aplicada à Oftalmologia. Oftalmologia em Foco. 2004;90:17-8.

20. Scheie HG, Albert DM. Distichiasis and trichiasis: origin and management. Am J Ophthalmol. 1966;61(4):718-20.

21. Yeung YM, Hon CY, Ho CK. A simple surgical treatment for upper lid trichiasis. Ophthalmic Surg Lasers. 1997;28(1):74-6.

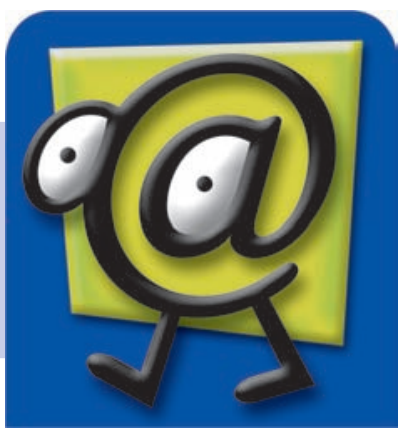

